Instituto Internacional de Investigación y Desarrollo Tecnológico Educativo INDTEC, C.A.

DOI: https://doi.org/10.29394/scientific.issn.2542-2987.2017.0.0.24.448-469

OAI-PMH: http://www.indteca.com/ojs/index.php/Revista Scientific/oai

\title{
Programa Institucional de Capacitación y Protagonismo Permanente. Formación Humanista desde el Vivir-Compartir
}

\author{
Autores: Ciro Hernández Valderrama \\ Instituto Universitario de Tecnología Puerto Cabello, IUTPC \\ cirohv@hotmail.com \\ Puerto Cabello, Venezuela \\ María Alejandra Hernández Domínguez \\ Instituto Universitario de Tecnología Puerto Cabello, IUTPC \\ marialeeducadora27@gmail.com \\ Puerto Cabello, Venezuela
}

\section{Resumen}

En la actualidad, instituciones educativas de cualquier nivel académico, se encuentran inmersas en un conglomerado de fenómenos científicotecnológicos y socioculturales, que los envuelve en un ambiente de dudas e insatisfacciones, por efecto el trabajo pedagógico parece haber perdido la brújula que lo guíe al encuentro con la plenitud interpersonal. Por ello, desde el área formación humanista de la Universidad Politécnica De Puerto Cabello, emerge el motivo pedagógico del presente programa. el mismo involucra la experiencia docente y observación directa. se enmarca en el paradigma cualitativo bajo la novedad acción-indagación-reflexión de tipo protagónico. atiende el proceso metodológico de investigar mientras se capacita. El proceso de diagnosticar, ejecutar y valorar, constituye una dinámica holística de interpretación y triangulación de información, proveniente de la observación participante y la discusión grupal. Mediante la exposición de contenidos temáticos en jornadas y sesiones de trabajo conjunto, se pretende entre otros aspectos: el ser reflexivo-crítico de la realidad docente-pedagogía, recuperar la disposición y apresto docente a compartir con alumnos, colegas y demás participantes de la agenda educativa, tomar conciencia del significado humano de la pedagogía y respeto a la dignidad que tiene el alumno, como ser humano semejante al profesor mismo.

Palabras clave: valores docentes; formación humanista; vivircompartir. 


\title{
Institutional Program of Training and Permanent Protagonism. Humanist Training from the Living-Sharing
}

\begin{abstract}
At present, educational institutions of any academic level are immersed in a conglomeration of scientific-technological and socio-cultural phenomena, which surrounds them in an environment of doubts and dissatisfactions, for the effect the pedagogical work seems to have lost the compass that guides it to encounter with the interpersonal fullness. Therefore, from the Humanist Training area of the Universidad Politécnica of Puerto Cabello, the pedagogical reason of this program emerges. It involves the teaching experience and direct observation. It is framed in the qualitative paradigm under the novelty actioninquiry-reflection of a leading role type. This program serves the methodological process of investigating while training. The process of diagnosing, executing and evaluating constitutes an holistic dynamic of interpretation and triangulation of information, derived from participant observation and group discussion. By means of the exposition of thematic contents in days and sessions of joint work, it is tried among other aspects: the reflective-critical being of the teaching reality-pedagogy, to recover the readiness and teaching preparation to share with students, colleagues and other participants of the educational agenda, become aware of the human meaning of pedagogy and respect for the dignity that the student has, as a human being similar to the teacher himself.
\end{abstract}

Keywords: teaching values; humanistic formation; living-sharing.

Date Received: 29-11-2016

Date Acceptance: 16-01-2017 


\section{Presentación Y Cosmovisión}

Los grupos humanos desde su constitución como tal, han evolucionado hasta la fecha, en supuestas ciudades modernas civilizadas, con alto nivel de progreso capitalista, científico-tecnológico, sin embargo, estamos presenciando la degradación mundial de lo ético-moral particularmente de los valores humanos, personales, laborales, colectivos. Nuestras instituciones universitarias no escapan de los efectos oprobiosos de estos fenómenos. Es evidente, en nuestros predios universitarios la presencia de fenómenos, tales como: deserción estudiantil, deficiente rendimiento académico, deprimida matrícula de bachilleres inscritos, ausentismo laboral y crítica formación profesional del egresado.

Sin embargo, la República Bolivariana de Venezuela transita a comienzos de siglo por profundas transformaciones, encaminadas hacia la fundación de un ciudadano y una ciudadana creativos y conscientes de su propio destino, con principios y valores de cooperación, unidad e integración, que garanticen dignidad y bienestar individual y colectivo, tal como está expreso en el Preámbulo y los artículos: 2, 3, 4, 102, 103 y 104 de la Constitución de la República Bolivariana de Venezuela (1999), lo cual sienta las bases en un proceso de educación integral, para todos y todas como fundamento de la transformación social, política, económica, territorial e internacional inspirado en el pensamiento de Simón Bolívar. De igual manera, la orientación humana, establece un Estado de justicia que consolide los valores de libertad, independencia, paz, solidaridad, bien común, convivencia, el derecho a la vida, al trabajo, a la cultura, a la educación y a la igualdad sin discriminación.

Desde esta cosmovisión se fundamentan los planteamientos teóricofilosóficos, conceptuales y metodológicos del área Formación Humanista, adscrita a la División de Docencia de la Universidad Politécnica de Puerto Cabello, toda vez que, se concibe como parte integrada y un elemento más 
del todo académico universitario. Es así, como la organización denominada universidad puede existir desde lo humano y axiológico en armonía plena; se evidencia que somos parte de un todo nacional, con un objetivo común derivado en la identidad del venezolano.

El Ministerio del Poder Popular Para la Educación Universitaria Ciencia y Tecnología de Venezuela, asume la responsabilidad de planificar, organizar, dirigir, coordinar, controlar corregir y reorientar el proceso educativo nacional, con el propósito de formar ciudadanos y ciudadanas útiles y virtuosos, que puedan construir una Patria donde, con la mayor suma de felicidad, paz y bienestar.

El ser humano que entiende el colectivo, según Hernández (2009) “...es el ser humano de la vida diaria, con todos los valores del buen vivir, siendo entre algunos de los más anhelados: la cortesía, solidaridad, honestidad, generosidad, responsabilidad, respeto a los demás y a los bienes ajenos..." (pág. 124), es decir, se procura conservar en la convivencia las dimensiones culturales de la experiencia humana.

Esto es, lo que da sentido al ser humano, es parte de las dimensiones humanas en el esquema de vida occidental. Es el estar, en el escenario continuo de interacciones, donde se dice que se hace cultura, política, educación, economía, ciencia.

En palabras del autor Hernández (2012) y para expresarlo en lenguaje cotidiano, si un maestro durante la clase, simultáneamente disfruta, se siente feliz, percibe un goce interno o sencillamente le agrada conversar con los alumnos. Esos alumnos van a incorporar espontáneamente la asignatura, el disfrute y el agrado, al espacio relacional consensuado con el profesor. Lo cual constituye, el instrumento de convivencia a través del cual, esos alumnos van a transformarse en adultos socialmente integrados con confianza en sí mismos, con capacidad de colaborar y de aprender cualquier cosa sin perder su conciencia ética. (pág. 15) 
De lo que se trata en la educación venezolana, es buscar nuevas formas de interactuar y conversar en el salón de clases, a fin de movilizar a los alumnos como actores de ese espacio social, hacia un cambio, orientado a fortalecer emociones, capacidades, sistematizar ideas e ideales en la lógica del amor y el bien común.

El sistema educativo constituye un proceso comparado con una cadena de vivencias, en la cual están contiguamente ubicados dos eslabones compartidos por el docente y el alumno. Por lo general, el eslabón correspondiente al profesor y a la función rectora que desempeña, es el más quebradizo y, cuando esto ocurre devienen los alejamientos y frustraciones.

Asimismo, se espera que en el proceso de interacción docente-alumnouniversidad, el pedimento constitucional sobre la reconocida moralidad e idoneidad académica del docente, trascienda los linderos de la inercia personal que lo sujeta, en pueriles certezas sobre su accionar pedagógico y lo catapulte a consolidar la unión con el otro eslabón y, ser innovador del propio espacio de interacción.

Desde este contexto, Hernández (ob.cit.) afirma, la docencia se define como actividad humana, consistente en un proceso sistemático de educar y enseñar; dentro del sistema educativo, es a todas luces tarea práctica. Por su parte, los docentes, son ciudadanos que se insertan y vinculan con la sociedad, colocándose en dialogo con sus tendencias, problemas, urgencias y dilemas. En esa relación se acompaña a los alumnos con la cultura y sociedad en la que viven y que les pertenece. (pág. 8)

En atención a lo expuesto, sobreviene la propuesta de programa institucional de capacitación y protagonismo permanente. Encuentro para la formación humanista desde el ser del vivir-compartir dirigido a alumnos, profesores, administrativos y personal obrero recurso humano de la Universidad. Las pretensiones, guarda pertinencia humana, con la necesidad de mejorar la gestión universitaria en sus dimensiones curriculares, 
académico-educativa y personal-social, a manera de, vivir conviviendo según lo establecido en el ordenamiento constitucional venezolano.

En medio de este contexto, es tácito preguntarnos ¿en qué hemos estado fallando?, ¿qué factores humanos y pedagógicos determinan estos fenómenos en nuestra institución universitaria?, ¿qué actitudes y racionalidades hay que conjugar para construir el éxito en la formación profesional del egresado de la Universidad Politécnica de Puerto Cabello?

\section{Discusión Argumental del Programa Institucional}

Es dentro de este horizonte de caos axiológico, y realidad presente en la institución educativa nacional y comunidades vecinas, que la propuesta adquiere importancia para la persona como ser sociocultural y humano, por cuanto de lo que se trata, es de introducir nuevas formas de pensar la pedagogía y la formación del ciudadano que viene de tales comunidades. Así lo plantea Hernández (2015) El vivir compartiendo supone el accionar del hombre, mediante un proceso de orden sistémico y experiencial, de vida personal-colectiva, que se construye en un contexto determinado. (pág. 12)

De igual manera, la propuesta se concibe como un programa de actividades en valores de convivencia, de aplicación teórico-práctico en jornadas y sesiones especiales, por su estructura organizada, expositiva, informativa y comunicacional, lo convierte en un hecho realizable en el tiempo y espacio, que permitirá capacitar para la construcción de cultura universitaria. Asimismo, apoyar epistemológicamente, otros proyectos de esta índole, en el sentido de orientar metodológicamente, la investigación pedagógica participante enmarcada en proyectos de revisión curricular.

Además, como aporte teórico, reforzará en los predios universitarios, el debate del campo axiológico, centrado en la unión docente-universidad, docente-docente, docente-alumno, docente-trabajador, docente-comunidad y la convivencia social planetaria; siguiendo desde luego, las matrices del 
novísimo Proyecto Nacional Simón Bolívar (2013-2019). Por otra parte, la universidad amerita del docente la capacitación para construir contextos cónsonos, diseñar estrategias didácticas particulares adaptadas a la realidad inmediata, que estimulen la creatividad de los alumnos. Como sabemos, a comienzos del tercer milenio, el ejercicio de la verdadera pedagogía parece comprometido, confronta estrujamientos provenientes de otras esferas del quehacer social, lo que trae como consecuencia dificultades para formar y capacitar al hombre.

Es imperativo que el docente se desprenda de la clase tautológica, de repetición de contenidos que año tras año transmite a sus alumnos; el acto pedagógico, más que una interacción, supone interrelaciones trascendentes, deslumbrantes, tendentes a fortalecer la creatividad, la racionalidad, la reflexión moral, de manera tal, que un docente debe ser protagonista activo, con la virtud de aprender aprendiendo con el alumno y, beneficiar el desarrollo de aptitudes.

El camino expedito para llegar a convivir en la transformación con el otro, es el de traspasar las barreras de la indiferencia y transitar por el imperativo de la conciencia, que es la dimensión humana de mayor ontología, desplegada en los discursos actuales sobre valores de más arraigo personal y social. Es este el sentido práctico que justifica la noción capacitación y protagonismo permanente.

En este contexto, el aporte teleológico del área formación humanista subyace en los protagonistas de la Universidad Politécnica de Puerto Cabello integrados a la vida en plena convivencia.

Entre otros: Brindar al estudiante en común acuerdo, herramientas que contribuyan a liberar los obstáculos cognitivos, emocionales y vocacionales que limitan la participación en la vida universitaria. Desarrollar proyectos de investigación, dirigidos a diagnosticar necesidades comunitarias y del estudiante a los fines de obtener, un conocimiento valido y confiable para la 
elaboración de planes y programas. Asesorar para el desarrollo de proyectos y planes. Apoyar a orientadores y docentes de institutos educativos a nivel medio y diversificado, cuya población estudiantil proceda de estratos bajos y tenga menor acceso a la Universidad.

Desde esta óptica, se justifica en la Universidad la existencia de un espacio de convivencia en la formación humana, que funcione de manera interrelacionada con otros espacios humanos de coordinación integral institucional.

\section{Sustento Teórico}

Los fundamentos teóricos, que sustenta la praxis del programa institucional de capacitación y protagonismo permanente desde el ser del vivircompartir, están relacionados con el aprendizaje, la comunicación y el comportamiento humano, veamos:

Por naturaleza biológica, somos de condición vivir compartir con el semejante; porque diluye entre todos: el nosotros, el vamos, el hemos, el estamos. Desde la visión epistémico-sistémico nada de lo que conocemos, pensamos y hacemos está suelto en lo social, todo es interdependiente. El saber cotidiano y la interpretación cercana del mismo, es exclusivo desde el contexto mismo y nuestro ángulo personal.

De igual forma, aprendemos en interrelación recíproca, a través del intercambio dialógico comunicacional, recursivo y hologramático. Por ello, nos reconocemos desde la dimensión cotidiana del ser; en consecuencia, la participación protagónica y vivencial constituye el modo cognitivo de las instituciones para resolver problemas de comunicación y tomar decisiones.

Conforme a estos postulados, el trabajo colectivo, concienzudo y sistemático de todos los involucrados en esta propuesta, fija como tarea, el éxito en su ejecución permanente, la renovación constante, y la innovación con nuevos elementos metodológicos, que reclamen emergentes realidades, ese 
debe ser el cometido inmediato. De igual manera, persiste el anhelo por lograr los valores universales plasmados en la novísima Constitución de la República Bolivariana de Venezuela (ob.cit.), y la Ley Orgánica de educación (2009); documentos de referencia universal a los que, remitimos nuestra conducta moral y orientarán la trascendencia en el tiempo, de las pretensiones aquí señaladas. Conforme e esta dimensionalidad el área formación humanista, fija la misión, visión y prospectiva social:

3.1. Misión: Cooperar desde los más sublimes sentimientos y emociones humanas en la formación de un ciudadano digno, en correspondencia con los más altos principios y valores de solidaridad e igualdad social. Toda vez, que desde la coordinación de la División de Docencia y Subdirección Académica subyace la entrega esmerada por el respeto y la aceptación del otro en la comunicación y convivencia.

3.2. Visión: Convertirse en un espacio de reseña institucional e interinstitucional, en la promoción de los principios y valores que constituyen la condición y el despliegue de la felicidad de la vida humana, en el entorno social universitario. Toda vez, que la trascendencia de la Formación Humanista avance al unísono con el interés institucional local, regional y nacional.

3.3. Prospectiva Social: Llegar a ser la universidad politécnica de mayor prestigio en la formación de líderes, que promulguen los fundamentos y valores humanos establecidos en La Constitución de la República Bolivariana de Venezuela. La prospectiva social se entiende como la proyección teleológica humanista de los actores universitarios y demás entes activos de la cosmovisión local y regional, para visionar un nuevo orden de ideas tendentes a la construcción del humanismo del siglo XXI. 


\section{Pretensiones}

Pretender sensibilizar a la comunidad universitaria en la construcción de su propia red axiológica y modo de compartido, a través de jornadas de capacitación, mediante encuentros humanos, es una labor ardua, que amerita de personas abnegadas por el bienestar del prójimo y de la propia institución; siendo que, las instituciones nacionales de formación educativa, transitan en medio de fenómenos humanos, constituidos en una compleja red de relaciones interpersonales, que opera en continua construcción y reconstrucción, de manera recursiva, del todo multidimensional a las particularidades de la vida personal y cotidiana. Es obvio que se trata de un trabajo laborioso, que debe ser planificado y valorado constantemente, a manera de lograr la metacognición en el intercambio de saberes y haceres, lo cual servirá para profundizar en futuras estrategias de formación.

Entre algunos valores humanos, institucionales, educativos, familiares, sociales y nacionales, a ser abordados estratégicamente, en el programa institucional de capacitación y protagonismo permanente destacan: el vivircompartiendo: el trabajo docente, el respeto y amor a Dios Todopoderoso, el respeto a la dignidad de la persona humana implícito en el alumnado, la solidaridad entre compañeros de labores, el respeto a la integridad física, la honestidad en el comportamiento, la responsabilidad en el trabajo, el amor por la unión familiar y los vecinos, el amor por el socialismo bolivariano, el amor por los valores socio-históricos y culturales que designan la identidad nacional venezolana.

De acuerdo con esto, Hernández (2012) aduce: el respeto a la dignidad del hombre constituye una herencia familiar, que se transmite desde la formación hogareña, por vivencias personales arraigadas en el sentir emocional de los actores sociales. (pág. 80) Por otra parte, se pretende reafirmar el amor por los símbolos patrios, el amor por los próceres de nuestra 
independencia republicana, el amor por el ideario del Padre de la Patria Simón Bolívar.

La formulación de estas pretensiones responde al contenido temático y de discusión, constituye una meta, que irá cobrando sentido y consolidación en la medida que avance el desarrollo de jornadas y sesiones de trabajo con los participantes. Visto de este modo y en atención a las prioridades institucionales en el ámbito de los valores académicos, socioeducativo y de formación profesional, se pretende en esta propuesta lograr:

\subsection{Pretensiones Generales}

- Reorientar la pedagogía de aula y planificación curricular.

- Resignificar la dignidad del docente en la autoexigencia profesional.

- Reacomodar la identidad-pertenencia con la institución universitaria.

- Integrar el protagonismo institucional universitario.

\subsection{Pretensiones Específicas}

- Construir reflexión moral para la autoexigencia profesional, en beneficio de la formación y capacitación del estudiante universitario.

- Capacitar al docente en comunicación pedagógica y didáctica.

- Construir espacios de reflexión, prevención de indiferencia docente, el sentido común y el sano juicio en las labores pedagógicas.

- Comprender que la enseñanza y aprendizaje de los valores institucionales de responsabilidad y puntualidad, constituye un espacio psíquico entrelazado con el conversar y el emocionar de sus miembros.

- Comprender que los valores de dignidad y convivencia, se generan en el espacio de relaciones e interacciones.

- Evidenciar como la racionalidad profesional, funciona para prevenir los antivalores y motivar el emocionar solidario en el escenario educativo. 
- Promover espacios para la convivencia familiar, desde la práctica del respeto a la dignidad del otro como legítimo semejante.

- Sensibilizar el comportamiento de identidad-pertenencia institucional, vinculado con el tratamiento preventivo de la desidia y aburrimiento laboral.

- Incorporar al docente a labores propias de organización y planificación curricular, ejecución de actividades administrativas, compromiso académico y pedagógico.

- Motivar el trabajo en equipo, mediante la invitación a protagonizar eventos deportivos, culturales, efemérides y onomásticos.

- Conectar recíprocamente a personal de dirección, en el funcionamiento curricular, pedagógico y académico de la institución.

Como se evidencia, el contenido semántico de las pretensiones y su aplicación, contribuirá dentro de la institución educativa, a capacitar para superar los problemas personales y colectivos en que nos encontramos inmersos; dado el conglomerado de fenómenos científico-tecnológicos y socioculturales, que nos envuelve, en un ambiente de dudas $e$ insatisfacciones, y en el que, programas de aplicación permanente como el presente, hace el papel de brújula orientadora, que guía los actores y objetivos institucionales hacia el logro en plenitud humana.

\section{Estrategia Cognitiva para la Comunicación en Valores}

La comunicación e información en un centro educativo, figura un espacio preponderante en el éxito de cualquier jornada de formación y capacitación colectiva. Es condición de los valores del hombre su naturaleza con el arraigo verbal, es allí, donde asisten al temperamento del hombre en sociedad, constituyen vigencia histórica y dinamizan el contexto humano de interacción e interrelación. Se propone el relato metafórico como estrategia 
comunicacional para reforzar y asistir las valoraciones pedagógicas, durante los conversatorios.

El lenguaje: permite describir lo observado, expresar lo sentido, lo pensado, habla de una realidad preexistente a los enunciados posibles sobre ella, cuenta cosas sobre la realidad que lo precede siempre. Garciandía (2011) afirma: El lenguaje no solo permite hablar sobre la realidad y como suceden las cosas, sino que además puede hacer que las cosas ocurran. (pág. 278) El lenguaje tiene pues, un carácter también ontológico, generador de ser.

Es a través de estrategias cognitivas de disertación y relato público como la metáfora, la analogía, la parábola, el cuento y la anécdota entre otros, que el programa de formación y capacitación tendrá el apoyo cognitivo y establecimiento de nuevas formas de pensar y esquemas mentales de vida y trabajo.

En este orden, la estrategia de compartir el festejo del onomástico entre los más cercanos al entorno pedagógico, construye aprehensión del compañerismo y arraigo en el trabajo docente.

¿Por qué la expresión metafórica? Según Hernández (2016), porque el individuo es esencialmente una entidad hablante, pensante y expresa su naturaleza subjetiva en lenguaje, dentro del sistema de sentidos de la vida cotidiana, de manera tal, que favorece la urdimbre el pensamiento con el lenguaje figurado. Promueve el descubrimiento, la estima, el esfuerzo, el trabajo y el estudio como fuentes de avance personal y colectivo. (pág. 10)

\section{Procedimiento y Contexto}

No sabemos cómo transcurre realmente un día en el escenario educativo de nuestra universidad, sólo sabemos cómo los vemos y lo observamos, en definitiva, cómo lo interpretamos, es decir, la realidad pedagógica en que estamos inmersos no es única, es multiversa que emerge de la multiplicidad de significaciones posibles, dependientes de múltiples 
observadores que sinérgicamente observamos nuestros entornos y contextos. Visto de este modo, Garciandía (ob.cit.) sostiene que vivimos en mundos interpretativos que conviven en un tiempo y espacio. Este planteamiento frustra al individuo en su pretendida búsqueda de la verdad ya que esta responde a una intensión que exige el ajuste entre lo que son las cosas y lo que decimos que son. (pág. 281).

Por ello, es que la propuesta nace de la experiencia docente-aula y, deriva en una participación constante de investigación educativa por el horizonte pedagógico. Mientras ocurren los encuentros para la reflexión con los participantes el investigador hace su trabajo de indagación e interpretación de información. En efecto, se trata de la búsqueda de actitudes, posturas personales, rasgos subjetivos, y temperamentales, entre nosotros para luego, reflexionar moralmente sobre éstos, e intentar transformar nuestra propia realidad institucional. El camino metodológico constituye en este caso, una dinámica recursiva de investigación-participación y participación-investigación.

Estaremos orientados por el paradigma cualitativo porque estamos vinculados con un centro educativo de formación humanista. Asimismo, la matriz epistémica del proceso cognitivo será la fenomenología y la hermenéutica caracterizada por abordar las vivencias concretas, tal como son interpretadas por los actores educativos. Bajo el visor acucioso y perspicacia interpretativa del investigador participante, cuya labor constante redundará sobre las manifestaciones de comportamiento durante los encuentros para la reflexión.

Los encuentros vivenciales están respaldados por la indagación- acción pedagógica de tipo participativo, por constituir un proceso constante de acciónobservación, reflexión y de acompañamiento protagónico entre los participantes. De esta manera, la retroalimentación lograda en lo grupos de discusión y mesas de trabajo hermenéutico, visualizan la formación y capacitación plasmadas en las pretensiones formuladas. 
Pretender alcanzar lo que está dicho en esta propuesta, comporta un desafío complejo, donde nos toparemos con problemas insospechados. Sin embargo, en aras de contribuir con el socialismo bolivariano afanosamente buscaremos nuevos saberes entre nosotros mismos. Esto significa, disponer de numerosos esfuerzos emocionales, personales, materiales, técnicos y tecnológicos.

Activar el desarrollo del programa institucional de capacitación y protagonismo permanente. Encuentro para la formación humanista desde el ser del vivir-compartir, implica disponer de un cronograma de encuentros 0 jornadas para la realización de: foros, video conferencias, películas, simposios de reflexión ético-moral, mesas de discusión, de participación mancomunada y solidaria. Se realizarán ponencias específicas de contenido didácticopedagógico orientado a sensibilizar el estado de ánimo, emerger la empatía y crear las condiciones mínimas para el trabajo proactivo y la sinergia individual y colectiva.

Estas actividades constituyen la fuente de recolección de información, dado que, las técnicas cualitativas respectivas, se basan en la discusión grupal, entrevistas y observación protagónica mediante encuentros personales en las aulas, departamentos, pasillos, biblioteca, áreas verdes, campo deportivo, entre otros espacios administrativos y académicos.

En cuanto al trazado de la propuesta, la misma supone un proceso de diagnóstico, ejecución y valoración, para los efectos, estos términos se entienden como momentos de un todo sistémico, de interrelación, observación participante y discusión grupal, en tanto que, las partes constitutivas son análogas e interdependientes y forman la unidad global estudiada. Dicho proceso configura círculos concéntricos, abiertos a la interrelación humana y a la diversidad de ideas y opiniones, con un movimiento que va del todo a las partes, y de las partes al todo, en una dinámica holística de análisis, interpretación y triangulación de información. 
En efecto diagnosticar implica, indagar nosotros mismos, a través de la observación participante y discusiones grupales con profesores, profesoras y alumnos, cuyo propósito subyacente, es el de recoger información pertinente para planificar actividades que respondan a las opiniones y sugerencias de los participantes. Para tal fin, en las sesiones de trabajo se aborda un conjunto de temas, relacionados con la problemática ya señalada y, se hace invitación a participar. Es en este momento de la investigación, y a través de la narrativa, que se activa la lectura hermenéutica de las necesidades y expectativas pedagógicas presentes en la institución.

La ejecución de la propuesta atañe al proceso de actuación, frente a la realidad institucional y pedagógica en el que estamos inmersos y, en el que podemos lograr reflexión moral y transformar el escenario; es el segundo momento, propicio para lograr interrelación con nuestro espacio académico, administrativo, pedagógico y personal-social, en el que construiremos racionalidad crítica a favor de modos de vida y esquemas de conocimiento. Corresponde en este espacio el inicio y cumplimiento de las acciones programadas.

El tercer momento atañe a valoración de rasgos personales, y asegurar sistematización de emergencias. Para abordar este espacio de relación, es preciso desarrollar discusión dialógica, entre iguales.

Con el pretendido de interactuar en formación integral humana, entre la universidad con las necesidades reales de la comunidad educativa local, se realizarán jornadas sociales abiertas e interactivas. Prevaleciendo para ello un trato de confianza con el ser humano, así como el respeto a su capacidad para afrontar con éxito la toma de decisiones y solución de problemas durante la vida. Una orientación coherente con esa confianza y respeto, son la clave de la proyección de la Universidad con la comunidad educativa para la vida en amor y libertad y por el camino de la felicidad. 
¿Por qué, valoración de rasgos? Comprendemos que la universidad es una institución, proveniente de la misma sociedad, siendo que, de manera recursiva ambas se reproducen, en consecuencia, somos parte constituyente del fenómeno sociocultural, de la formación y capacitación de las comunidades $\mathrm{y}$, nadie más que nosotros mismos para auto-valorarnos, coe-valorarnos, hetero-valorarnos y conocer nuestro propio escenario.

Cabe señalar en este espacio, la preponderancia y acepción que le conferimos al término valorar en vez del término evaluar, en el sentido que, nuestras pretensiones, obedecen más a la comprensión de las interioridades y valores del hombre $y$, por ende, dicho término guarda en lo cognitivoontológico relación tácita. Por ello, de lo que se trata es de priorizar la integración de saberes y haceres en la multidiversidad de ideas y opiniones, formar al hermano para la reflexión en valores, emancipación y trascendencia en rasgos humanos. Lo cual representa un soporte cualitativo de la evaluación empírica y experimental basada en contenidos.

Para que esta búsqueda de la unidad en lo diverso se constituya en un hecho objetivo y concreto, no debemos tener preferencias a priori por ninguna información ideas y sentimientos, nuestra posición a asumir será crítica, de consenso y de buena voluntad.

En tal sentido, es válida la programación de jornadas de formación o capacitación durante el transcurso de actividades académicas regulares, orientadas a mejorar tanto la calidad de vida emocional, en nuestros espacios laborales y socio-comunales, como reforzar la ética socialista, en aras de construir la patria humana soñada por nuestros Libertadores.

La autovaloración en el fragor de la discusión grupal y la observación participante, constituye un proceso continuo de triangulación, rectificación, reajuste y reimpulso, realizado por los mismos participantes y las personas que fungen de motivadores y coordinadores de las actividades. De igual manera, la institución como ente rector que adscribe y viabiliza la propuesta, 
tiene la prerrogativa de indagar y sugerir innovaciones a los procesos de formación y capacitación, en función de la participación y creatividad.

Al concluir cada jornada, se analizará e interpretará sistemáticamente, el nivel de asimilación, formación y capacitación. Esta valoración debe profundizar en los progresos alcanzados, los registros de asistencia, la autorecomendación y aspectos cualitativos de la personalidad. La autovaloración sirve para informar sobre el grado de cumplimiento de las actividades y su aplicabilidad; asimismo, sensibiliza y motiva para aumentar la capacidad de trabajo, construir sinergia y acercar empáticamente a las personas.

En una propuesta institucional como esta, que pretende en lo metodológico la doble intención de investigar mientras se capacita, el desempeño del investigador docente en el aula, debe estar desprendido de imperativos personales y de la presunta autoridad o poder sobre el sujeto que aprende. Lo ideal sería que la ontología que encierra el ser docente, priorice la influencia que pueda ejercer el participante sobre sí mismo.

La formación de hábitos y consolidación de valores educativos no puede ser pensada si no es en la relación con el otro, con las dimensiones cognitivas y afectivas. El sujeto se forma siempre en la relación con otro, nunca se forma aislado, de manera tal, que en una relación de aula se le debe al participante en formación, la posibilidad de autonomía, de expresión, de opción y de elección en función de su propio trayecto a partir de sus necesidades. Estas dimensiones deben estar presentes como aspectos epistemológicos en el investigador.

Entre las técnicas e instrumentos a ser usados para recoger información, están: observación participante y los grupos de discusión, mediante diarios y notas de campo, procedimiento mediante el cual se apunta la información al momento de ser observada, tanto por los participantes, como los investigadores y motivadores. El uso de cuestionarios para solicitar opinión a estudiantes, análisis de documentos y la conversación abierta. Como 
instrumentos de registro se usarán las grabaciones, videos y sesiones fotográficas.

El procedimiento analítico y de interpretación a emplear, durante y después de las reuniones con los participantes, es la triangulación. Como ya fue referido el análisis de la información comienza desde el mismo momento en que se registra la información en los instrumentos respectivos. El fundamento teórico implícito en la noción de triangulación según Elliot (2000), es el de integrar informes y observaciones sobre situaciones similares, realizados desde diferentes ópticas y perspectivas, para compararlos y contrastarlos. Este movimiento de interpretación, es el eje fundamental de la propuesta, porque conduce a la producción de constructos y elementos de juicio, con tendencia a clarificar problemas y orientar decisiones.

Se prevé que, de la triangulación, interpretación y contrastación del proceso hermenéutico, emerjan convergencias y con ello la manifestación de posturas y actitudes personales, que servirán de información clave para hacer ajustes y reacomodos de forma y contenido en las próximas jornadas.

\section{A modo de conclusión}

En el proceso pedagógico institucional, el vivir-compartir transcurre mediante una especie de florecimiento constante de circunstancias naturales, que van consecuencialmente forjando un dominio de acciones, muy propio del escenario y que le da connotación humana, en ese espacio de coordinaciones recurrentes, entre las cuales el amor, lenguaje y el conversar forman una red de valoraciones, constituyendo lo humano del sujeto. De allí que, el Programa Institucional de Capacitación y Protagonismo Permanente, desde el ser del Vivir compartir, está abierto a nuevos aportes y reflexiones de los docentes a partir de sus experiencias.

Se vaticina que esta propuesta sea inspiradora como alternativa para ofrecer apoyo pedagógico. El programa es extensivo a otros espacios 
educativos, y se aplique no solamente en nuestra institución universitaria, sino que pueda desarrollarse en otras instituciones y otros espacios sociales de las comunidades más cercanas a nuestra institución.

\section{Referencias}

Constitución de la República Bolivariana de Venezuela (1999). Gaceta Oficial № 5453 Extraordinario. Caracas, Venezuela.

Elliot, J. (2000). El cambio educativo desde la investigación-acción. (3 ed.) Madrid: Morata.

Garciandía, J. (2011). Pensar sistémico. Una introducción al pensamiento sistémico. Bogotá: Pontificia Universidad Javeriana.

Hernández, C. (2009). El sentido de lo humano en el contexto educativo venezolano. Desde la teoría de Maturana. ARJÉ. Revista de Postgrado FACE-UC. 3(4), 121-136. Venezuela.

Hernández, C. (2012). El trabajo docente y el respeto a la dignidad en el contexto de la educación media venezolana. Tesis Doctoral. Cabudare. Venezuela: Universidad Fermín Toro.

Hernández, C. (2016). Valores y Lenguaje. Revista Scientific, 1(2), 341-362. Recuperado de:

https://doi.org/10.29394/scientific.issn.2542-2987.2016.1.2.19.341-362 Ley Orgánica de Educación (2009). Gaceta Oficial N 5929 Extraordinario. 15 de agosto. Caracas, Venezuela.

Proyecto Nacional Simón Bolívar (2013-2019). Gaceta Oficial de la República Bolivariana de Venezuela No 6.118 Extraordinario, 4 de diciembre. Venezuela. 


\section{Ciro Hernández Valderrama}

e-mail: cirohv@hotmail.com

Nacido en el Estado Trujillo, Venezuela. Licenciado

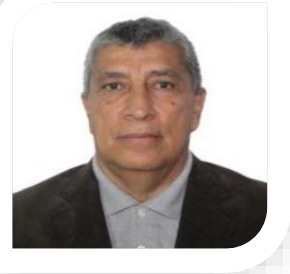
en educación, mención: Orientación egresado de la Universidad de Carabobo. Maestría en Educación Básica (Universidad Nacional Experimental Rómulo Gallegos). Doctor en Ciencias de la Educación (Universidad Fermín Toro). Postdoctor en Estudios Libres (UFT). Cuya agenda constituye un proceso sistematizado de investigación, producción, análisis y comunicación de conocimientos. Así mismo, la epistemología como una reflexión filosófica en torno al estado del arte científico, que pretende dar cuenta de los modos de pensar y conocer los estilos de pensamiento, teorías y prácticas en campos disciplinares específicos. Profesor Asociado, adscrito a Departamentos de Mecánica Térmica y Automotriz en la Universidad Politécnica de Puerto Cabello. Venezuela, coordinador de proyectos dirigidos a orientación con valores y de convivencia socio-comunitaria. Ponente en congresos académicos. Participante con artículos en revistas científicas. Dictaminador en revistas científicas: ARJE, CIENCIAS DE LA EDUCACIÓN, EDUCARE UPEL IPB., INVESTIGACIONES INTERACTIVAS COBAIND, SCIENTIARUM, EDUCARE UNA, Jurado en trabajo de ascenso: UNIPAP, IUTPC. Tutor de tesis UC. Investigador socioeducativo, en la dimensión ético-moral del hombre. Certificado en Programa de Estímulo a la Innovación e Investigación. PEII convocatoria 2015-2016, Investigador A-1, adscrito al Observatorio Nacional de Ciencia Tecnología e Innovación (ONCTI). 


\section{María Alejandra Hernández Domínguez \\ e-mail: marialeeducadora27@gmail.com}

Natural del Estado Carabobo, Venezuela. Licenciada

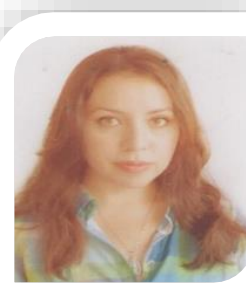
en educación, mención: Idiomas Modernos, egresada de la Universidad de Carabobo. Maestría en Lectura y escritura, bajo la línea de investigación: Comprensión de textos y recepción significativa del discurso textual, procesos cognitivos, metacognitivos, sociales y psicoafectivos. (Universidad de Carabobo). Profesora Instructor. Designada al Departamento de Mecánica Automotriz en la Universidad Politécnica de Puerto Cabello Venezuela. Docente Investigador en semántica de estrategias didácticas, para el aprendizaje y evaluación del fenómeno educativo universitario. Apoyo técnico a proyectos dirigidos en orientación con valores y de convivencia social. Aspirante al Programa de Estímulo a la Innovación e Investigación (PEII) 2016-2017).

El contenido de este manuscrito se difunde bajo una Licencia de Creative Commons ReconocimientoNoComercial-Compartirlgual 4.0 Internacional 